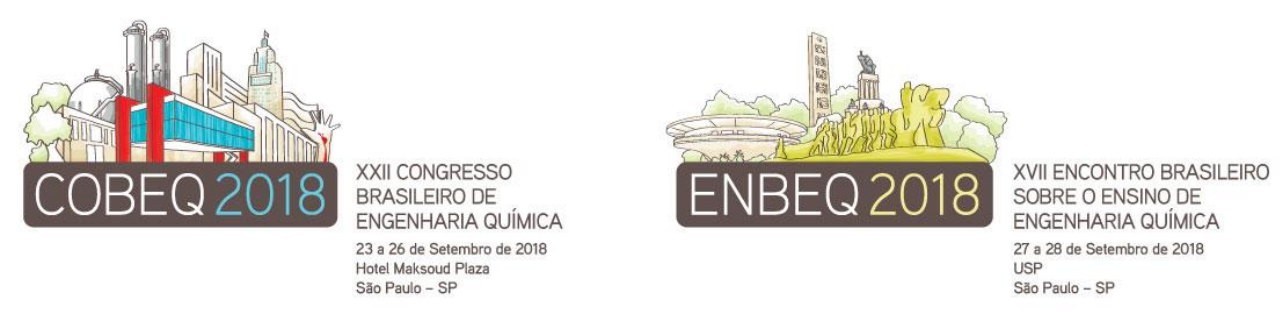

\title{
FERMENTAÇÃO ALCOÓLICA EXTRATIVA IN SITU EM BATELADA ALIMENTADA USANDO BIORREATOR COLUNA DE GOTAS
}

\author{
LEMOS DA ${ }^{1}$, SONEGO JLS ${ }^{2}$, CRUZ AJG $^{3}$ e BADINO AC ${ }^{3}$ \\ ${ }^{1}$ Universidade Federal do Triângulo Mineiro, Instituto de Ciências Tecnológicas e Exatas, \\ Departamento de Engenharia Ambiental \\ ${ }^{2}$ Universidade Federal do Oeste da Bahia, Centro Multidisciplinar de Luís Eduardo \\ Magalhães \\ ${ }^{3}$ Universidade Federal de São Carlos, Departamento de Engenharia Química \\ E-mail para contato: diegolemos18@yahoo.com.br
}

\begin{abstract}
RESUMO - Nos processos industriais de produção de etanol, o acúmulo de etanol no caldo de fermentação inibe o crescimento celular e sua própria produção, resultando em baixa produtividade em produto. Uma forma de contornar esse efeito de inibição é o emprego da fermentação extrativa, na qual o etanol é removido do caldo de fermentação ao longo do processo. O objetivo do presente trabalho foi avaliar a produção de etanol em fermentação extrativa no modo batelada alimentada, usando ácido oleico como agente extrator em um biorreator coluna de gotas. Foi usado um modelo matemático para a fermentação extrativa de modo a prever o perfil das variáveis em estudo. $O$ modelo híbrido de Andrews-Levenspiel foi capaz de descrever a cinética do processo, prevendo fielmente o comportamento da fermentação extrativa. Fazendo uso de simulações, foi possível prever as condições para a realização de uma fermentação extrativa contendo aproximadamente $260,0 \mathrm{~kg} \cdot \mathrm{m}^{-3}$ de substrato, obtendo-se ao final uma concentração de etanol de $14,2^{\circ}$ GL, um valor $34 \%$ superior a uma fermentação convencional não extrativa.
\end{abstract}

\section{INTRODUÇÃO}

As fermentações extrativas são utilizadas para remover o etanol do caldo fermentativo ao longo do processo de produção, contornando a inibição pelo produto e a baixa produtividade que normalmente ocorrem nas fermentações convencionais. Neste contexto, a fermentação extrativa líquido-líquido tem um grande potencial. A incorporação de um agente extrator para remoção in situ é uma ferramenta poderosa para aliviar o efeito de concentrações elevadas de etanol (DAFOE e DAUGULIS, 2014). Outra preocupação durante a fermentação alcoólica são os efeitos inibitórios causados pelo substrato sobre a levedura, que devem ser evitados controlando a concentração de substrato na dorna em valores inferiores a $160,0 \mathrm{~kg} \cdot \mathrm{m}^{-3}$, pois essa situação pode levar à diminuição da velocidade de fermentação e do crescimento da levedura (ZHANG et al. 2015). Uma maneira de contornar este problema é o uso da fermentação em batelada alimentada, que permite regular a concentração de substrato na dorna tendo um grande efeito no processo. 


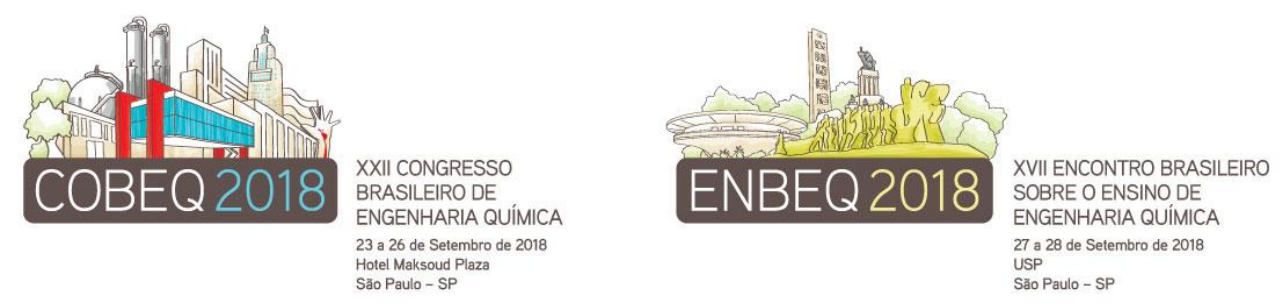

O objetivo do presente trabalho foi avaliar a produção de etanol em fermentação extrativa no modo batelada alimentada, usando ácido oleico como agente extrator em um biorreator coluna de gotas. Foi usado um modelo matemático para a fermentação extrativa de modo a prever o perfil das variáveis em estudo e compará-los aos dados obtidos experimentalmente.

\section{MATERIAIS E METODOLOGIA}

\subsection{Procedimento Experimental - Batelada Alimentada}

As fermentações em batelada alimentada foram conduzidas em biorreator tipo torre operado como uma coluna de gotas (BCG) (LEMOS et al., 2017). Como agente extrator foi utilizado ácido oleico. A composição do meio de cultivo consistiu de $\left(\mathrm{em} \mathrm{kg} \cdot \mathrm{m}^{-3}\right)$ : sacarose (açúcar cristal comercial) (FC: 190,0 e FE: 260,0), $\mathrm{KH}_{2} \mathrm{PO}_{4}(5,60), \mathrm{MgSO}_{4} .7 \mathrm{H}_{2} \mathrm{O}(1,40)$, extrato de levedura $(6,80)$ e ureia $(5,32)$. $\mathrm{O}$ pH inicial do caldo de fermentação foi ajustado para 4,6 com adição de ácido clorídrico $(1 \mathrm{M})$.

Na fermentação alcoólica convencional (FC), inicialmente um volume de inóculo de 0,6 L $\left(30 \% \mathrm{v} . \mathrm{v}^{-1}\right)$, contendo $50 \mathrm{~g} . \mathrm{L}^{-1}$ de levedura em massa seca foi previamente hidratado por 30 min em $0,5 \mathrm{~L}$ de água. Foi alimentado ao biorreator, $1,4 \mathrm{~L}$ de meio de cultura (substrato) na vazão de $0,28 \mathrm{~L} \cdot \mathrm{h}^{-1}$, totalizando $5 \mathrm{~h}$ de enchimento. O biorreator estava equipado com um sistema de circulação de caldo com vazão de $10 \mathrm{~L} \cdot \mathrm{h}^{-1}$ para assegurar uma mistura adequada do meio reacional. Após a inoculação, a levedura foi aclimatizada durante $15 \mathrm{~min}$ antes da remoção da primeira amostra. A temperatura foi mantida a $34^{\circ} \mathrm{C}$ e amostras de $10 \mathrm{~mL}$ foram retiradas a cada hora para análises. O volume do meio aumentou linearmente com o tempo.

Nos ensaios de fermentação extrativa (FE), seguiu-se o mesmo procedimento descrito para a fermentação convencional (FC), sendo que ao se atingir concentrações próximas de 40,0 $\mathrm{kg} \cdot \mathrm{m}^{-3}$ de etanol e volumes acima de $1 \mathrm{~L}$ (garantindo a relação máxima caldo/solvente de 1:1) era inserido o ácido oleico e a agitação do meio passava a ser realizada pela recirculação do solvente através do caldo. Neste momento, o ácido oleico era inserido pela base do biorreator e pela diferença de densidade ele passava através do caldo (em forma de gotas) até a parte superior, de onde ele era bombeado para um novo ciclo até que estivesse saturado de etanol. $\mathrm{O}$ volume total de solvente empregado foi de $5 \mathrm{~L}$, sendo que este volume foi alimentado de maneira fracionada - $1 \mathrm{~L}$ por vez - nos tempos de fermentação 3, 6, 8, 10 e $11 \mathrm{~h}$ de processo. Estas condições previamente simuladas com os parâmetros obtidos a partir da fermentação convencional tinham o intuito de aumentar a produção e finalizar a fermentação extrativa com concentrações de etanol próximas às obtidas nas fermentações convencionais, mesmo quando empregadas altas concentrações de substrato $\left(260,0 \mathrm{~kg} \cdot \mathrm{m}^{-3}\right)$. O tempo de enchimento foi de 6 h.

\subsection{Modelagem Matemática}

$\mathrm{Na}$ fermentação extrativa (FE), durante a fase de enchimento do biorreator $(\mathrm{F} \neq 0)$, considerando a geração do produto (etanol) associada ao crescimento celular, o modelo é representado por quatro equações diferenciais relativas aos balanços de massa para as células $(\mathrm{X})$, substrato $(\mathrm{S})$, etanol $(\mathrm{E})$ e variação de volume devido à vazão de alimentação $(\mathrm{F})$ : 


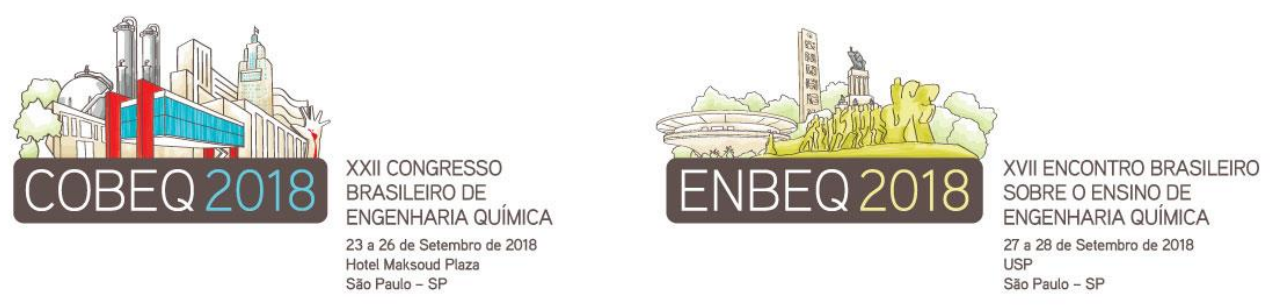

$\frac{d C_{X}}{d t}=\mu \cdot C_{X}-C_{X} \cdot \frac{1}{V} \cdot F$

$\frac{d C_{S}}{d t}=\left(C_{S F}-C_{S}\right) \cdot \frac{1}{V} \cdot F-\frac{1}{Y_{X / S}} \cdot \mu \cdot C_{X}$

$\frac{d C_{E}}{d t}=\frac{Y_{E / S}}{Y_{X / S}} \cdot \mu \cdot C_{X}-C_{E} \cdot \frac{1}{V} \cdot F$

$\frac{d V}{d t}=F$

onde $\mathrm{C}_{\mathrm{X}}$ é a concentração celular $\left(\mathrm{kg} . \mathrm{m}^{-3}\right), \mu$ é a velocidade específica de crescimento celular $\left(\mathrm{h}^{-1}\right), \mathrm{C}_{\mathrm{S}}$ é a concentração de substrato limitante $\left(\mathrm{kg} \cdot \mathrm{m}^{-3}\right)$ (açúcares redutores totais), $\mathrm{C}_{\mathrm{SF}}$ é a concentração de substrato na alimentação $\left(\mathrm{kg} \cdot \mathrm{m}^{-3}\right), \mathrm{C}_{\mathrm{E}}$ é a concentração de etanol $\left(\mathrm{kg} \cdot \mathrm{m}^{-3}\right), \mathrm{Y}_{\mathrm{X} / \mathrm{S}}$ é o coeficiente de rendimento celular $\left(\mathrm{kg}_{\mathrm{X}} \cdot \mathrm{g}_{\mathrm{S}}{ }^{-1}\right), \mathrm{Y}_{\mathrm{E} / \mathrm{S}}$ é o coeficiente de rendimento em etanol $\left(\mathrm{kg}_{\mathrm{E}} \cdot \mathrm{kg}_{\mathrm{s}}{ }^{-1}\right)$ e $\mathrm{V}$ o volume biorreator (L).

A velocidade específica de crescimento celular foi representada pelo modelo cinético de Andrews-Levenspiel, que considera a inibição tanto pelo substrato quanto pelo produto:

$\mu=\mu_{\max } \cdot \frac{C_{S}}{K_{S}+C_{S}+\frac{C_{S}^{2}}{K_{I S}}} \cdot\left(1-\frac{C_{E A}}{C_{E \max }}\right)^{n}$

onde $\mu_{\max }$ é a velocidade específica de crescimento celular máxima $\left(\mathrm{h}^{-1}\right), \mathrm{K}_{\mathrm{S}}$ é a constante de saturação $\left(\mathrm{kg} \cdot \mathrm{m}^{-3}\right), \mathrm{K}_{\mathrm{IS}}$ é a constante de inibição do substrato $\left(\mathrm{kg} \cdot \mathrm{m}^{-3}\right), \mathrm{C}_{\text {Emax }}$ é a concentração máxima de etanol tolerada $\left(\mathrm{kg} . \mathrm{m}^{-3}\right)$ e n é uma constante adimensional. $\mathrm{O}$ valor de $\mathrm{C}_{\mathrm{EA}}$ representa a concentração de etanol no caldo de fermentação (fase aquosa) no BCG, que é inferior à do processo convencional $\left(\mathrm{C}_{\mathrm{E}}\right)$. Isto ocorre devido à distribuição instantânea nas fases aquosa $\mathrm{e}$ orgânica da massa de etanol produzido, mesmo quando as células de levedura consumiram a mesma quantidade de substrato, devido à menor concentração de etanol $\left(\mathrm{C}_{\mathrm{EA}}\right)$ que as leveduras são expostas, causando menor inibição pelo produto.

\section{RESULTADOS E DISCUSSÃO}

Na Tabela 1 é possível comparar os desempenhos dos dois processos fermentativos. A concentração de etanol no caldo ao final da fermentação extrativa foi igual a $72,4 \mathrm{~kg} \cdot \mathrm{m}^{-3}$ $\left(9,2^{\circ} \mathrm{GL}\right)$. Este valor está muito próximo aos valores obtidos nas destilarias brasileiras (8$10^{\circ} \mathrm{GL}$ ). Isto significa que o vinho obtido nas fermentações extrativas com mosto concentrado pode ser enviado para a etapa de destilação sem comprometer a eficácia do processo.

Tabela 1 - Comparativo dos desempenhos das fermentações convencional (FC: $\mathrm{Cs}_{\mathrm{F}}=190,0$ $\left.\mathrm{kg} \cdot \mathrm{m}^{-3}\right)$ e extrativa $\left(\mathrm{FE}: \mathrm{Cs}_{\mathrm{F}}=260,0 \mathrm{~kg} \cdot \mathrm{m}^{-3}\right)$ em batelada alimentada com extração in situ.

\begin{tabular}{cccc}
\hline Variável & Unidades & $\mathrm{FC}$ & $\mathrm{FE}$ \\
\hline Cso & $\mathrm{kg} \cdot \mathrm{m}^{-3}$ & $190,5 \pm 2,4$ & $257,0 \pm 0,8$ \\
CEA final no caldo & $\mathrm{kg} \cdot \mathrm{m}^{-3}$ & $83,6 \pm 2,1$ & $72,4 \pm 2,8$ \\
C $_{\text {E }}$ total no final da fermentação & $\mathrm{kg} \cdot \mathrm{m}^{-3}$ & $83,6 \pm 2,1$ & $112,0 \pm 2,8$ \\
${ }^{\circ} \mathrm{GL}$ & $\% \mathrm{v} \cdot \mathrm{v}^{-1}$ & 10,6 & 14,2 \\
Etanol removido & $\% \mathrm{~m} \cdot \mathrm{m}^{-1}$ & - & 35,38
\end{tabular}

A Figura 1 apresenta os resultados obtidos para fermentação extrativa FE, realizada com uma concentração total de substrato alimentado de $260,0 \mathrm{~kg} \cdot \mathrm{m}^{-3}$, tempo de enchimento de $6 \mathrm{~h}$ 


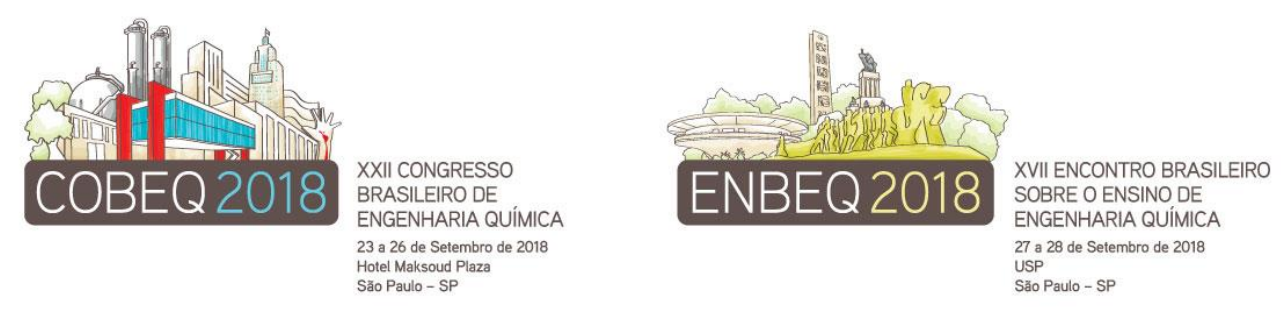

e com início do processo de extração em 3 h de fermentação, quando a concentração de etanol alcançou o valor de $33,6 \mathrm{~kg} \cdot \mathrm{m}^{-3}$. Neste caso, a extração se iniciou com um valor de etanol um pouco abaixo do esperado pela simulação, porém foi adequado para a manutenção do etanol a baixas concentrações durante o processo. Sob estas mesmas condições, a concentração máxima de substrato no caldo de fermentação alcançou o valor de $135,9 \mathrm{~kg} \cdot \mathrm{m}^{-3} \mathrm{e}$ a concentração máxima de etanol de $72,4 \mathrm{~kg} \cdot \mathrm{m}^{-3}$. O tempo de enchimento de $6 \mathrm{~h}$ e o início da extração em $3 \mathrm{~h}$ resultou em uma fermentação com consumo total do substrato em $14 \mathrm{~h}$, com uma produção de etanol $34 \%$ superior à convencional. Sonego et al. (2018) relatam que em condições similares, porém com retirada de etanol por arraste com $\mathrm{CO}_{2}$, obteve-se uma concentração máxima de etanol de $72,0 \mathrm{~kg} \cdot \mathrm{m}^{-3}$, mostrando que a extração líquido-líquido pode ser tão eficiente quanto outros tipos de processos extrativos. Neste caso, no qual foram removidos $35,4 \%$ de etanol, a energia gasta para separar o etanol do ácido oleico por flash é menor à da destilação convencional (JASSAL et al., 1994), além do ácido oleico recuperado poder ser utilizado numa próxima extração, não causando danos ao meio ambiente com seu descarte após um único uso. Além disso, haveria economia no consumo de vapor e uma diminuição na geração de vinhaça, diminuindo assim os custos do processo.

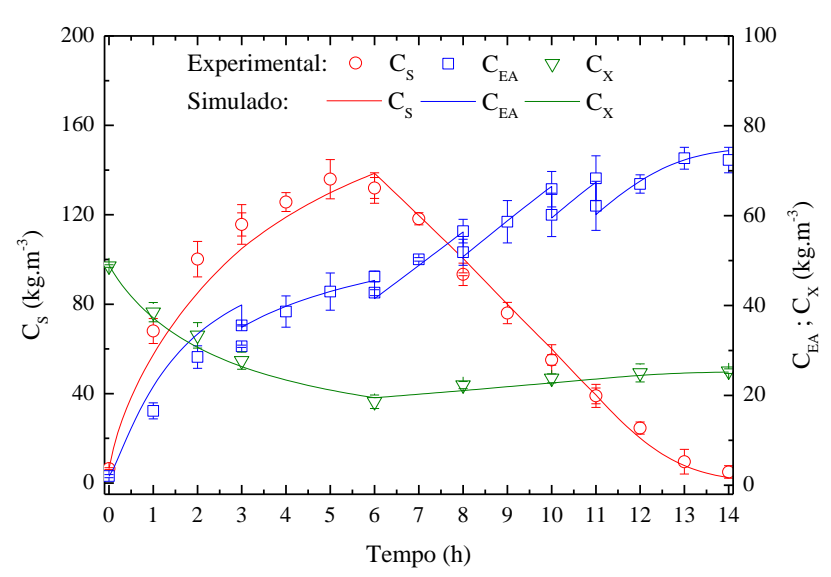

Figura 1 - Comparativo de dados experimentais e simulados para fermentação extrativa em BCG com ácido oleico e $260 \mathrm{~kg} \cdot \mathrm{m}^{-3}$ de concentração total de substrato alimentado (FE).

\section{REFERÊNCIAS}

DAFOE, J.T. E DAUGULIS, A.J. In situ product removal in fermentation systems: improved process performance and rational extractant selection. Biotechnology Letters, 36, 443-460, 2014.

JASSAL, D.S.; ZHANG, Z.; HILL, G.A. In situ extraction and purification of ethanol using commercial oleic acid. Canadian Journal of Chemical Engineering, 72, 822-827, 1994.

LEMOS, D.A.; SONEGO, J.L.S.; CRUZ, A.J.G.; BADINO, A.C. In situ extractive ethanol fermentation in a drop column bioreactor. Journal of Chemical Technology and Biotechnology, 2017.

SONEGO, J.L.S.; LEMOS, D.A.; CRUZ, A.J.G.; BADINO, A.C. Optimization of Fed-Batch Fermentation with in Situ Ethanol Removal by $\mathrm{CO}_{2}$ Stripping. Energy \& Fuels, 32, 954-960, 2018.

ZHANG, Q.; WU, D.; LIN, Y.; WANG, X.; KONG, H.; TANAKA, S. Substrate and product inhibition on yeast performance in ethanol fermentation. Energy \& Fuels, 29, 1019-1027, 2015. 